\title{
Liga Acadêmica interdisciplinar de Saúde Mental: ampliando a formação e as práticas no campo da atenção psicossocial
}

John Victor dos Santos Silva ${ }^{1}$ (D), Claudio José dos Santos Júnior ${ }^{2}$ (D), Larissa Dandara Lima dos Santos ${ }^{3}$ (D), Vívian Mayara da Silva Barbosa ${ }^{4}$ (D), Thyara Maia Brandão² (D), Mara Cristina Ribeiro² (D)

\begin{abstract}
RESUMO
Fundamentos: A Liga Acadêmica Interdisciplinar de Saúde Mental (LAISME) constitui um espaço de conhecimento e reflexão crítica na área de saúde mental, visando ampliar a formação e as práticas na atenção psicossocial. Objetivo: relatar a experiência de estudantes universitários na criação, organização e realização de atividades através de uma liga de saúde mental como estratégia de ampliar o conhecimento sobre o campo da atenção psicossocial. Método: trata-se de um trabalho descritivo do tipo relato de experiência sobre uma liga de saúde mental pertencente a uma universidade pública do nordeste do Brasil, no período de 2016 a 2019. Resultado: a partir das atividades desenvolvidas, a Liga favoreceu um maior aprendizado e aproximação com os cenários e atores da saúde mental, além de viabilizar o desenvolvimento de aptidões como a produção científica, a organização de eventos e o trabalho junto à comunidade em ações de promoção da saúde. Conclusão: a experiência de integrar a LAISME proporcionou aos estudantes uma maior aproximação com o campo da atenção psicossocial, além de favorecer o desenvolvimento de habilidades na realização de eventos e trabalho em equipe. Ressalta-se, ainda, a importância de fomentar estratégias como as ligas acadêmicas enquanto forma de efetivação das vivências de ensino, pesquisa e extensão e da convivência interdisciplinar.
\end{abstract}

Palavras-chave: Saúde mental, Serviços de saúde mental, Educação superior, Currículo.

\section{INTRODUÇÃO}

As Ligas Acadêmicas são conhecidas como entidades estudantis sem fins lucrativos, vinculadas às Instituições de Ensino Superior (IES) e que contam com a tutoria ou coordenação de professores ou profissionais da instituição. Elas podem ser constituídas de diversas formas: pela categoria profissional, sendo para estudantes de um único curso; por uma especialidade, dentro de algumas categorias profissionais; ou mais abrangentes, sendo de uma área de conhecimento geral, tendo participação de estudantes de diversos cursos e categorias profissionais, como é o caso da Liga Acadêmica Interdisciplinar de Saúde Mental ${ }^{1}$.

Essas ligas constituem uma opção para os estudantes aprofundarem o conhecimento a respeito de determinados temas e como estratégia de desenvolver diversas atividades práticas em determinadas áreas do conhecimento. Essas atividades desenvolvidas pelas ligas geralmente são aulas ou cursos de capacitação e são conferidas por vários professores ou profissionais que dominam ou atuam na área de interesse da liga acadêmica. Algumas ligas favorecem a realização de práticas na assistência em diversos serviços de saúde, além de contribuírem para o desenvolvimento de pesquisas e ações na comunidade ${ }^{2}$.

Por ser uma atividade extracurricular, as ligas acadêmicas abrigam em seus objetivos o exercício da teoria, prática e pesquisa sobre a temática proposta de forma indissociada e complementar ao aprendizado curricular. No desenvolvimento das diversas atividades desses eixos, o estudante é favorecido também ao ensaio de uma vivência profissional interdisciplinar que se mos-

\footnotetext{
Universidade de São Paulo (USP). Escola de Enfermagem de Ribeirão Preto. Programa de Pós-graduação em Enfermagem Psiquiátrica, Ribeirão Preto (SP), Brasil.

Universidade Estadual de Ciências da Saúde de Alagoas. Centro de Ciências da Saúde, Maceió (AL), Brasil.

Santa Casa de Misericórdia de Maceió. Maceió (AL), Brasil.

Universidade Federal de Alagoas. Escola de enfermagem, Maceió (AL), Brasil.
} 
tra essencial aos cenários que compõem seu contexto de aprendizado, sobretudo, na saúde mental ao contribuir para a assistência nessa área ${ }^{3}$.

A motivação para participar de uma Liga Acadêmica pode surgir por diversos fatores. Pode ser pela curiosidade em conhecer ou se aproximar de determinados assuntos, para aprofundamento em algumas temáticas, pela oportunidade de prática em serviços de saúde, ou até mesmo pela pouca ênfase e aprofundamento em determinadas áreas nas disciplinas e cursos de graduação, o que permite que o estudante possa complementar sua formação através das atividades desenvolvidas na liga, proporcionando uma nova experiência ${ }^{4}$.

Além de todas essas oportunidades que as ligas podem proporcionar aos estudantes, elas podem ser espaços para que os mesmos exercitem o trabalho em equipe, o respeito, a liderança, a resolução de problemas e contribuições maiores para o meio acadêmico, como a realização de eventos, seminários, para discutir com a comunidade acadêmica e, em geral, as questões que vêm sendo debatidas e aprendidas no decorrer das atividades realizadas ${ }^{5}$.

Uma das áreas pouco exploradas na maioria dos cursos de graduação é a saúde mental, o que motiva estudantes de diversas instituições a participarem de ligas acadêmicas, projetos de extensão, eventos ou cursos como forma de aproximação e aprofundamento na temática e garantir, assim, uma maior prática e conhecimento sobre os cuidados às pessoas que necessitam dessa atenção ${ }^{6}$.

Desta forma, o objetivo do presente estudo é relatar a experiência de estudantes universitários na criação, organização e realização de atividades através de uma liga de saúde mental como estratégia de ampliar o conhecimento sobre o campo da atenção psicossocial.

\section{MÉTODOS}

Trata-se de um trabalho descritivo do tipo relato de experiência, que favorece reflexões sobre as práticas e vivências desenvolvidas no âmbito profissional e acadêmico, sobretudo no contexto da interdisciplinaridade vivenciada no SUS ${ }^{7}$. Acrescidas ao relato, foram utilizadas informações referentes à liga em questão.
O presente estudo traz informações sobre a criação, organização e ações realizadas pela Liga Acadêmica Interdisciplinar de Saúde Mental (LAISME), que é uma entidade estudantil, sem fins lucrativos, vinculada à Pró-Reitoria de Extensão (PROEX) de uma Universidade do nordeste brasileiro, e que tem como finalidade promover atividades e ações de extensão universitária no campo da Saúde Mental e Atenção Psicossocial na perspectiva da Reforma Psiquiátrica e tendo como referencial as Políticas Públicas de saúde e de educação.

Anualmente são disponibilizadas, em média, vinte vagas para o ingresso na liga, que se dá a partir da participação do aluno no curso introdutório e, posteriormente, na realização da prova de seleção, onde os alunos com maior média de pontuação tornam-se membros. As vagas da LAISME podem ser preenchidas por estudantes de cursos de graduação da área de saúde e/ou áreas afins pertencentes à instituição proponente e demais instituições de ensino superior de Alagoas. Os novos membros, ao ingressarem na liga, recebem um cronograma e começam a participar das reuniões científicas que acontecem na Universidade uma vez a cada mês com carga horária de oito horas, e que conta com a participação de professores e profissionais para realizar capacitações sobre as temáticas da saúde mental e saúde em geral.

Os membros também participam das ações de educação em saúde em escolas e instituições, e também visitas aos serviços de saúde mental, além dos eventos realizados pela LAISME ao Iongo do ano, como seminários, encontros e simpósios nas datas comemorativas, como o dia da luta antimanicomial, setembro amarelo, dia mundial da saúde mental, entre outros.

O estudante que ingressa na liga fica um período de doze meses do ciclo vigente, sendo condicionado às regras de permanência e realização das atividades para permanecer vinculado a esta na qualidade de membro. Após o término do ciclo vigente, os membros recebem certificação pelo tempo e são selecionados novos membros para o ciclo seguinte. Para recebimento de certificação, os participantes precisam atingir $75 \%$ de frequência nas atividades desenvolvidas ao longo do ciclo. 
Os materiais utilizados pela LAISME nas suas reuniões e atividades são próprios da Universidade ou comprados através dos fundos arrecadas pelos eventos realizados pela liga. A LAISME ainda conta com apoio de instituições de saúde, ONGs e de outras Ligas para o desenvolvimento de suas atividades e ações.

As atas das reuniões ordinárias e extraordinárias, os projetos e relatórios dos eventos realizados bem como outros instrumentos de registro de atividades desenvolvidas pela liga foram utilizados como fontes de dados na construção deste relato. Os materiais foram ordenados de forma cronológica e, a partir de repetidas leituras, foram estabelecidos os pontos descritos no trabalho.

\section{RESULTADO}

\section{A criação da LAISME}

A LAISME surgiu através do interesse de estudantes da Universidade em questão em conhecer mais sobre o campo da Saúde Mental e da Atenção Psicossocial. A criação da liga possibilitou aos membros um contato maior com a temática, paralelamente ao que se estava aprendendo na Universidade. Em alguns cursos, saúde mental é uma disciplina pequena ou um assunto integrado em outras disciplinas e nem sempre contempla a magnitude do trabalho desenvolvido e das políticas existentes sobre os serviços, o trabalho e a atenção em saúde mental. A abertura da liga aproximou os estudantes com os profissionais da área e abriu os horizontes sobre a formação nesta temática.

A LAISME foi fundada em julho de 2016, através da criação do seu estatuto e cadastro na Pró-Reitoria de Extensão, e tendo como membros diretores fundadores estudantes dos cursos de enfermagem, medicina, terapia ocupacional e fonoaudiologia. $O$ primeiro evento realizado pela LAISME foi o I Curso Introdutório, que aconteceu em novembro de 2016, com tema "A Atenção Interdisciplinar na Saúde Mental Infantil" e que deu início ao ciclo 2016-2017 da liga, contando com a participação de noventa e cinco pessoas entre estudantes e profissionais de diversas áreas e formações, que estiveram presente e prestigiaram o evento, dando início ao estabelecimento da LAISME. O evento foi direcionado para todos que tivessem interesse na temática, principalmente os estudantes das instituições de ensino superior, pois através do curso seria feita a prova de seleção para integrar novos membros nas atividades da liga.

Figura 1: Logo utilizada pela Liga Acadêmica Interdisciplinar de Saúde Mental.

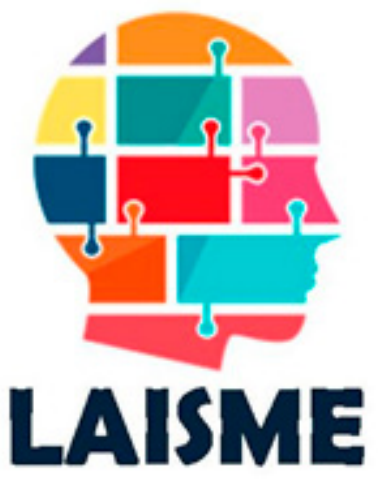

Fonte: https://www.instagram.com/laismeuncisal/.

Após a realização do curso introdutório, a LAISME organizou a prova de seleção para estudantes que iriam compor o quadro de novos membros. A prova foi realizada também em novembro de 2016, ofertando 20 vagas para estudantes de todas as áreas. Através da primeira prova, foram selecionados estudantes dos cursos de Enfermagem, Fonoaudiologia, Fisioterapia, Terapia Ocupacional, Psicologia, Medicina, Serviço Social, Direito, Comunicação Social/Jornalismo e Biologia, onde passaram um ano desempenhando atividades e ações na liga. A LAISME preza sempre pela formação interdisciplinar, entendendo que para se trabalhar em saúde mental é preciso ter a contribuição de vários profissionais da saúde e de outras áreas que desempenham papel importante no cuidado em saúde mental.

\section{Organização da Liga e Ações desenvolvidas}

Após o processo de seleção os novos membros, com os membros diretores (membros fundadores), começaram a se articular para organizar e realizar diversas atividades pela liga. As atividades tiveram início em fevereiro de 2017, quando começaram as reuniões científicas.

Em 2017 foram realizadas dez reuniões científicas, sendo uma por mês, onde os membros 
contaram com a participação de professores e profissionais ministrando aulas, oficinas e capacitações sobre os mais diversificados temas em saúde mental, como Reforma Psiquiátrica, Políticas Públicas em Saúde Mental, Saúde Mental na Atenção Básica, Matriciamento em Saúde Mental, Dependência Química, População em Situação de Rua, Redução de Danos, Rede de Atenção Psicossocial,
Centros de Atenção Psicossocial, Transtornos Mentais, Prática do Autocuidado, Práticas Integrativas e Complementares em Saúde, entre outros.

Além de profissionais docentes, algumas reuniões foram lideradas e organizadas pelos próprios membros, favorecendo o protagonismo dos próprios estudantes na articulação e construção do conhecimento.

Tabela 1: Número de membros da LAISME por curso de graduação e ciclo.

\begin{tabular}{lcc}
\hline & \multicolumn{2}{c}{ Número de membros } \\
\cline { 2 - 3 } Descrição do curso do membro & $\begin{array}{c}\text { Ciclo } \\
\text { Graduação em Biologia }\end{array}$ & $\mathbf{2 0 1 8 - 2 0 1 9}$ \\
\hline Graduação em Comunicação Social/Jornalismo & 1 & - \\
Graduação em Direito & 1 & - \\
Graduação em Enfermagem & 1 & - \\
Graduação em Fisioterapia & 3 & 4 \\
Graduação em Fonoaudiologia & 1 & 2 \\
Graduação em Medicina & 2 & 3 \\
Graduação em Psicologia & 1 & 2 \\
Graduação em Serviço Social & 4 & 3 \\
Graduação em Terapia Ocupacional & 3 & 3 \\
Total & 3 & 22 \\
\hline
\end{tabular}

Fonte: Elaborado pelos autores, 2020.

A liga também realizou, em 2017, uma visita técnica a um Hospital Escola de atenção psiquiátrica do Estado que pertence à Universidade. O objetivo da visita foi que os membros pudessem conhecer a assistência psiquiátrica no hospital para que fosse possível fazer um comparativo entre o campo da psiquiatria, que tem o hospital psiquiátrico no centro da assistência, e o cuidado nos serviços pós-reforma psiquiátrica que preconizam o cuidado inserido dentro na comunidade, como é o exemplo dos Centros de Atenção Psicossocial (CAPS). A visita foi um momento bastante rico que proporcionou aos estudantes uma reflexão sobre os dois modelos de assistência à pessoa com transtorno ou em sofrimento mental.

Ao longo do ciclo 2017-2018 a LAISME também foi protagonista de diversos eventos na área da saúde mental no estado. Em 2017 foram realizados três eventos: o I Encontro sobre Saúde Mental, Direitos Humanos e Luta Antimanicomial, que foi em alusão ao 18 de maio, dia Nacional da Luta Antimanicomial; o I Encontro sobre Prevenção ao Suicídio, que aconteceu para discutir as questões sobre suicídio durante o Setembro Amarelo; e o I
Simpósio do Dia Mundial da Saúde Mental, cujo objetivo foi trazer a percepção do autocuidado para todos, entendendo que existem diversos fatores internos e externos que podem colocar qualquer pessoa em situação de sofrimento mental.

A organização desses eventos foi feita por todos os membros da liga. Todos colaboraram em todo o processo de cada evento, com atividades de divulgação, captação de recursos, convidando os palestrantes, estabelecendo comunicação com a comunidade externa que desejasse participar dos eventos, com a ergonomia dos locais do evento, e diversas atividades. Esses eventos proporcionaram que os estudantes exercitassem o trabalho em grupo, em equipe, além de inseri-los em diversos cenários que proporcionaram o desenvolvimento de competências pessoais e coletivas.

Os membros da liga também participaram de diversos eventos locais, regionais e nacionais, levando a experiência da liga para os eventos em forma de apresentação de trabalhos científicos. Foram apresentados trabalhos no $1^{\circ}$ Congresso de Saúde Mental do estado, onde dois membros diretores compuseram uma mesa redonda sobre a extensão 
em saúde mental; no VII Congresso Acadêmico da Universidade, onde os membros tiveram a oportunidade de fazer uma exposição sobre as atividades desenvolvidas pela liga; no IV Congresso Brasileiro sobre Saúde Mental e Dependência Química, em João Pessoa, onde um grande grupo da liga participou ativamente e apresentando trabalhos científicos.

Essa participação da liga em outras atividades e eventos contribui para que os estudantes possam compartilhar suas experiências e vivências na liga e também para compreender a percepção de outros estudantes sobre a área, favorecendo uma rica troca de conhecimentos.

\section{Quadro 1: Eventos realizados pela LAISME de} 2016 a 2019.

\begin{tabular}{ll}
\hline Eventos realizados pela LAISME & Ano \\
\hline I Curso Introdutório da LAISME & 2016 \\
$\begin{array}{l}\text { I Encontro sobre Saúde Mental, Direitos Huma- } \\
\text { nos e Luta Antimanicomial }\end{array}$ & 2017 \\
I Encontro sobre Prevenção ao Suicídio & 2017 \\
I Simpósio do Dia Mundial da Saúde Mental & 2017 \\
II Curso Introdutório da LAISME & 2018 \\
II Encontro sobre Saúde Mental, Direitos Huma- & 2018 \\
nos e Luta Antimanicomial & 2018 \\
$\begin{array}{l}\text { II Encontro sobre Prevenção ao Suicídio } \\
\text { II Simpósio do Dia Mundial da Saúde Mental }\end{array}$ & 2018 \\
II Encontro de Enfermagem em Saúde Mental & 2019 \\
III Curso Introdutório da LAISME e III Encontro & 2019 \\
de Prevenção ao Suicídio & \\
\hline
\end{tabular}

Fonte: elaborado pelos autores, 2020.

A LAISME também realizou algumas atividades e reuniões com outras ligas da UNCISAL, a exemplo da Liga Acadêmica de Educação em Saúde (LAEDS) e a Liga Acadêmica de Saúde Pública (LASP). Essa interação com as outras ligas da instituição fortalece o discurso de integralidade que o Sistema Único de Saúde (SUS) preconiza, onde todos devem estar envolvidos no processo de promoção da saúde.

Após a conclusão do ciclo 2017-2018, alguns membros fundadores e diretores se desligaram das atividades da LAISME, o que levou a diretoria a selecionar, dentre os membros que ingressaram, alguns para fazerem parte do quadro dos membros diretores, que são responsáveis por toda organização, efetivação e gerenciamento das atividades. Ao final do ciclo, os demais membros receberam certificação pelo ciclo que permaneceram e finalizaram suas atividades com a liga. Esse período com os estudantes favoreceu o crescimento mútuo, tanto para os membros diretores, que permanecem ainda trabalhando na liga e para os membros que passaram um ano contribuindo para que as atividades e eventos da LAISME pudessem ser realizados.

No início de 2018 a LAISME começou o planejamento do seu calendário para as atividades do ciclo 2018-2019, que iniciou com a organização do II Curso Introdutório, realizado em maio de 2018, com tema "Atual Panorama da Saúde Mental: Para onde estamos caminhando?", com o objetivo de realizar nova prova de seleção para outros estudantes ingressarem na liga e contribuir nas novas atividades, ações e eventos. A prova de seleção foi realizada também em maio de 2018 e selecionou estudantes dos cursos de Enfermagem, Terapia Ocupacional, Fisioterapia, Medicina, Serviço Social e Psicologia. Em junho de 2018, os membros da LAISME participaram do $2^{\circ}$ Congresso de Saúde Mental, apresentando trabalhos.

Figura 2: Membros da LAISME. Na esquerda estão os membros do ciclo 2017-2018 e na direita os membros do ciclo $2018-2019$.

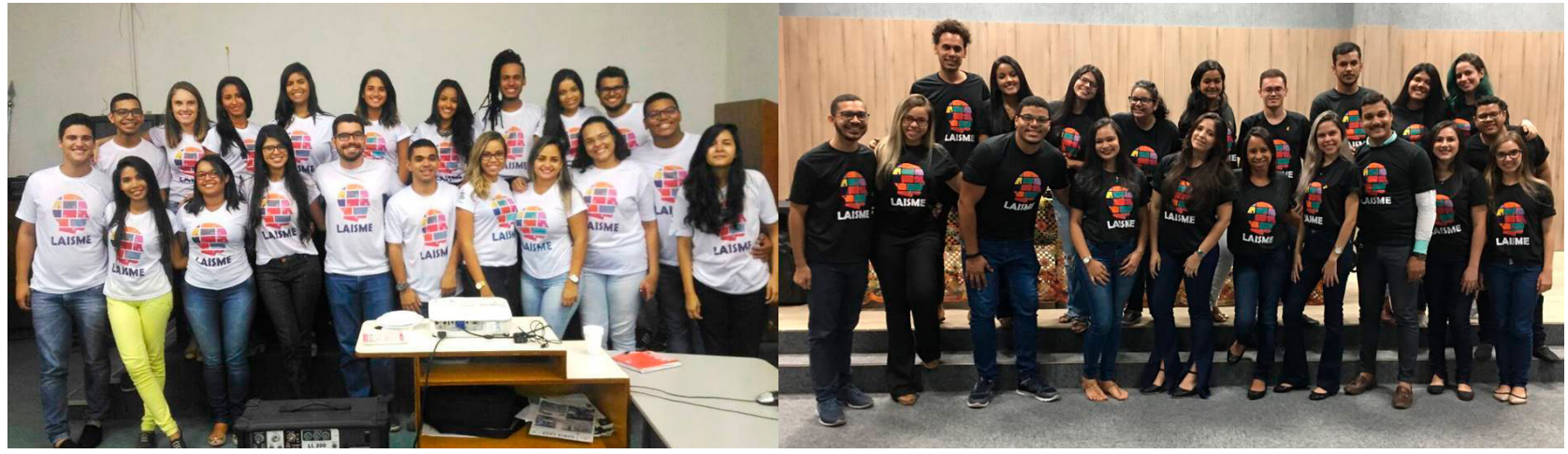

Fonte: arquivo dos autores, 2020. 
As atividades do novo ciclo começaram em julho de 2018, contando com a colaboração de todos os novos membros para o planejamento do novo ciclo. Ao decorrer do ano letivo de 2018, com a colaboração da equipe diretora e dos membros, foram realizadas as segundas edições dos eventos já realizados pela LAISME no ano anterior.

Em 2019, além dos eventos, foi organizada a primeira edição do Encontro de Enfermagem em Saúde Mental, específico para estudantes e profissionais enfermeiros, técnicos e auxiliares de enfermagem. Além disso, os membros e diretoria contribuíram e participaram do 30 Congresso Alagoas de Saúde Mental, através de oficinas e também apresentação de trabalhos.

Ao longo dos três anos de existência, a LAISME realizou diversas atividades, ações e eventos e oportunizou que estudantes, fundadores e os que permaneceram na liga durante os ciclos, tivessem contato com profissionais e serviços de saúde pública, outros estudantes de diversas instituições para uma rica troca de experiências e vivências, permitindo o protagonismo dos estudantes em diversos cenários, estimulando o trabalho em equipe de forma totalmente interdisciplinar.

\section{DISCUSSÃO}

A criação, organização e o desenvolvimento de ações através da LAISME, enquanto atividade complementar e paralela às atividades curriculares da graduação, viabilizou um contato mais abrangente com a área, possibilitou o desenvolvimento do ensino e da pesquisa, efetivando ações de extensão, promoveu o estabelecimento de vínculos entre estudantes, professores, profissionais e comunidade, e permitiu um cenário de integração e aprofundamento acerca da Reforma Psiquiátrica e de seus pilares, assim como contribuiu para a educação permanente e para a consolidação e o estímulo ao trabalho interdisciplinar.

Organizar os eventos desenvolvidos pela LAISME foi um desafio para todos os envolvidos, pela necessidade de conhecer um pouco sobre a assistência em saúde mental e a rede de serviços na cidade. Para isso, os estudantes entraram em contato com professores e profissionais no intuito de saber sobre os principais assuntos que poderiam ser abordados no evento, o que começou a oportunizar os convites para que esses profissionais e professores ministrassem algumas atividades com os membros da liga.

Diante das inúmeras mudanças que circundam a formação em saúde, as atividades de extensão são ótimas oportunidades para contribuir de forma significativa na complementação curricular dos estudantes. Um grande exemplo disso são as Ligas Acadêmicas, que acompanham essas mudanças no ensino ${ }^{8}$.

Do ponto de vista do ensino formal na área de saúde, há um evidente esforço para a modificação do modelo tradicional de organização do cuidado ao usuário e a comunidade, historicamente centralizados na doença e na figura do atendimento hospitalar. Foi na busca de alcançar esse perfil transformador que os diversos cursos superiores desta área editaram suas Diretrizes Nacionais Curriculares (DNC), de modo a propor, de forma explícita, mudanças no modelo de formação, na perspectiva de aproximar o perfil de competências dos profissionais de saúde às necessidades dos serviços, das comunidades e dos processos de trabalho ${ }^{9,10}$.

É nesse sentido que as DCN atuais dos cursos da área de saúde trazem como eixo norteador uma formatação curricular pautada nas necessidades de saúde mais frequentes da comunidade, sugerindo desenvolvimento de atitudes voltadas para a cidadania e valorizando o emprego de metodologias que privilegiem a integração entre 0 ensino, a pesquisa, a extensão e a assistência ${ }^{9,10}$.

Alguns autores ${ }^{11}$ chamam atenção para a necessidade de se promover práticas de ensino, pesquisa e extensão nas instituições de ensino superior, capazes de favorecer novas atitudes dos futuros profissionais em relação à assistência à saúde mental. Diante dessas reflexões, percebe-se a importância da adoção de diferentes percursos de formação e do protagonismo das metodologias ativas e complementares na construção do processo ensino-aprendizagem dos futuros profissionais de saúde, como é o caso da constituição de ligas acadêmicas, a exemplo da Liga Acadêmica Interdisciplinar de Saúde Mental aqui relatada. 
Outros autores ${ }^{12}$ destacaram a importância das ligas acadêmicas para a formação em saúde, enfatizando a qualificação profissional e a ampliação do olhar acerca do processo de cuidado favorecida com a atuação desses grupos - qualidades tão necessárias ao trabalho em Saúde Mental.

A proposta de contribuir com a aproximação de estudantes de graduação com o segmento da saúde mental, uma área ainda pouco visada como campo de trabalho por parte dos discentes da área de saúde em geral é cumprida pela liga. Esse aspecto é compartilhado alguns autores ${ }^{13}$ que afirmam que a participação dos alunos em ligas acadêmicas aumenta o interesse dos estudantes pelo seguimento na área, aproximando os acadêmicos com o dia a dia e com as funções desenvolvidas pelos profissionais em suas áreas de atuação.

O estímulo à interação ativa com a comunidade, o fortalecimento do vínculo da formação acadêmica com as necessidades sociais, a implementação de atividades didáticas visando trabaIhar a criatividade, a autoaprendizagem, a criticidade e o pensamento científico foram aspectos trabalhados ao longo das ações da LAISME.

A LAISME favoreceu maior aprendizado e aproximação de estudantes de graduação de diversos cursos da saúde e áreas correlatas, com aplicações práticas, e vivências na área de saúde mental, além de proporcionar o estreitamento de vínculos e o conhecimento acerca dos componentes da rede de atenção psicossocial regional, o desenvolvimento de aptidões como a produção científica e a organização de eventos e o trabalho junto à comunidade para a promoção da saúde.

Essa variabilidade de oportunidades é responsável por somar habilidades ao estudante e de viabilizar o desenvolvimento de novas competências, corroborando com outros autores ${ }^{3}$ que reforçam que as ligas não se limitam apenas no objetivo de aprofundar o conhecimento em determinada área, agregando valores à formação acadêmica e pessoal e contribuições à sociedade, seja pela disponibilização de um profissional mais capacitado para atuação nos serviços, seja pelo próprio desenvolvimento de ações de promoção de saúde e de transformações sociais executadas pelas ligas acadêmicas.
A área da saúde mental é conhecida por ser um dos ramos da área de saúde mais arraigados por pré-conceitos formulados pela comunidade em geral. Um estudo ${ }^{14}$ realizado com estudantes participantes de extensão em saúde mental mostrou que a participação dos alunos contribuiu na desconstrução e reformulação de conceitos formulados até os primeiros contatos com as pessoas com sofrimento mental e seus familiares e ampliou o conhecimento dos participantes sobre essa área. Nessa mesma perspectiva, as atividades da LAISME também oportunizaram uma nova concepção de saúde mental e de atendimento nesse campo de trabalho, perpassando pela abordagem de bases políticas e legais à organização das redes de atenção.

As abordagens da Saúde Mental e da Atenção Psicossocial à luz dos princípios da Reforma Psiquiátrica e tendo como referencial as políticas públicas de reestruturação da rede assistencial em saúde mental nos sistemas de saúde do Brasil realizadas pela Liga em suas atividades, apresentam-se em plena sintonia com o que trazem alguns autores sobre a importância da formação profissional, reorientação de posturas e reorganização do processo de formação em saúde para efetivação dos princípios políticos, ideológicos e práticos do ideário antimanicomial ${ }^{15,16}$.

Sabe-se que os diversos serviços de saúde mental dentro da RAPS no âmbito do SUS também são responsáveis pela formação de diversos estudantes e profissionais principalmente da saúde e também nas demais áreas, qualificando pessoas para o trabalho, assistência e cuidado das pessoas com transtornos mentais ${ }^{16,17}$.

Esses serviços possuem equipes compostas por profissionais de diversas formações, atuando através da perspectiva da interprofissionalidade $e$ interdisciplinaridade, permitindo que os estudantes que têm a oportunidade de realizar práticas, sejam através de aulas ou projetos de extensão, ampliem a construção do conhecimento na área de saúde mental, principalmente na perspectiva na interprofissionalidade ${ }^{18,19}$.

Ademais, a atuação interdisciplinar da Liga, viabilizada pela integração dos vários cursos de graduação da área de saúde, permitiu a apropriação prática de conceitos como o de responsabi- 
lização pelo cuidado, amplitude do ser humano, importância da formação de equipes, promoção de saúde, necessidade de criação de vínculo terapêutico e integralidade da assistência.

Do mesmo modo, menciona-se a contribuição do contato precoce com os profissionais que integram a equipe multiprofissional da RAPS, responsável por viabilizar a troca de conhecimento dos alunos com a equipe sobre as funções e atividades desenvolvidas e por viabilizar a integração ensino-serviço e o contato com os dispositivos da RAPS.

\section{CONCLUSÃO}

O presente trabalho constituiu um marco inicial na análise das contribuições da LAISME, sendo que outros estudos, mais analíticos, poderão ser desenvolvidos para verificar a percepção dos estudantes, profissionais e comunidade contemplada com as ações desenvolvidas pela Liga, na tentativa de verificar o impacto dessa iniciativa para a formação acadêmica, benefícios gerados para sociedade e outros.

As contribuições advindas da experiência aqui relatada reforçam a importância das instituições de ensino superior trabalhar a tríade ensino-pesquisa-extensão e, especificamente, a extensão em saúde mental, pois, como demonstrado, esta traz grandes contribuições para os estudantes e comunidade envolvida, sendo mediadora de uma formação em saúde mental complementar de qualidade e atrelada aos ditames preconizados para a formação do profissional de saúde contemporâneo.

Uma limitação do estudo foi a pouca disponibilidade de publicações dos últimos cinco anos acerca da temática para enriquecer e ampliar a análise do trabalho. As atividades desenvolvidas têm agregado valor à formação dos futuros profissionais da saúde e saúde mental, com paradigmas superados e com aptidões que só o ensino em sala de aula seria incapaz de contemplar.

\section{REFERÊNCIAS}

1. Silva DT. A evolução do processo ensino-aprendizagem através da implantação da liga acadêmica. Revista
Rede de Cuidados em Saúde [Internet]. 2014 [citado em 20 jan, 2020];8(2):1-5. Disponível em: http:// publicacoes.unigranrio.edu.br/index.php/rcs/article/ view/2277/1131.

2. Gutierre M, Gutierre R, Curiati JA. A liga de meditação e saúde na educação, prevenção e terapêutica de profissionais de saúde e pacientes. Rev. Med. (São Paulo) [Internet]. 2019;98(2):152-4. DOI: 10.11606/issn.16799836.v98i2p152-154.

3. Silva A, Flores O. Ligas Acadêmicas no Processo de Formação dos Estudantes. Rev. bras. educ. med., Rio de Janeiro, v. 39, n. 3, p. 410-417, Sept. 2015. DOI: 10.1590/1981-52712015v39n3e02592013.

4. Moreira COF, Dias MSA. Diretrizes Curriculares na saúde e as mudanças nos modelos de saúde e de educação. Abcs Health Sciences [Internet]. 2015;40(3):300305. DOI: 10.7322 /abcshs.v40i3.811.

5. Magalhães EP, Rechtma R, Barreto V. A liga acadêmica como ferramenta da formação em Psicologia: experiência da LAPES. Psicologia Escolar e Educacional [Internet]. 2015; 9(1):135-141. DOI: $10.1590 / 2175-$ 3539/2015/0191813.

6. Rosa RPF, Andrade ALF, Oliveira SP, Silva AGL, Ferreira AM, Inácio JS et al. Construindo saberes no trabalho em saúde mental: experiências de formação em saúde. Interface-Comunicação, Saúde, Educação [Internet]. 2015;19(supl.1):931-940. DOI: 10.1590/180757622014.0730 .

7. Weber A, Tombini LHT, Colliselli $L$, et al. Vivências e estágios na realidade do SUS (VER-SUS) e a formação profissional em saúde: relato de experiência. Revista Eletrônica de Extensão, Florianópolis-SC. 2016; 13(23):112-122. DOI: $10.5007 / 1807-0221.2016 \mathrm{v} 13 \mathrm{n} 2$ $3 p 112$.

8. Oliveira TC. Liga de Emergência Da UFC: relato de experiência de um projeto de extensão universitária. Revista Brasileira de Extensão Universitária [Internet]. 2017;8(2):83-89. DOI: 10.24317/23580399.2017v8i2.4972.

9. Brasil. Ministério da Educação. Resolução no 3, de 7 de novembro de 200. Institui Diretrizes Curriculares Nacionais do Curso de Graduação em Enfermagem. Brasília, DF, 2001.

10. Brasil. Ministério da Educação. Resolução CNE/CES n० 3, de 20 de junho de 2014. Institui Diretrizes Curriculares Nacionais do Curso de Graduação em Medicina e dá outras providências. Brasília, DF, 2001.

11. Olmos CEF, Rodrigues J, Lino MM, Lino MM, Fernandes JD, Lazzari Daniele Delacanal. Ensino de Enfermagem Psiquiátrica e Saúde Mental face aos currículos brasileiros. Rev. Bras. Enferm. [Internet]. 2020; 73(2): e20180200. DOI: 10.1590/0034-7167-2018-0200.

12. Cavalcante ASP, Vasconcelos MIO, Lira GV, Henriques TLM, Albuquerque INM, Ribeiro GP et al. As Ligas Acadêmicas na Área da Saúde: Lacunas do Conhecimento na Produção Científica Brasileira. Rev. Brasil. de 
educ. méd [Internet]. 2018;42(1):197-204. DOI: 10.1590/1981-52712018v42n1rb20170081.

13. Araújo RF, Cotta BSS, Resende LS, Alvarenga RPC, Magalhães LL, Kanadani FN. A contribuição na disseminação do conhecimento de Oftalmologia da primeira Liga Acadêmica de Belo Horizonte. Medicina (Ribeirao Preto Online). 2019;52(4):329-37. Disponível em: 10.11606/ issn.2176-7262.v52i4p329-337.

14. Mendes A, Marques M, Monteiro A, Barroso T, Quaresma $M$. Educação em enfermagem de saúde mental e psiquiatria no curso de licenciatura em enfermagem. SMAD Rev Eletrônica Saúde Mental Álcool Drogas [Internet]. 2018;14(2):73-83. DOI: 10.11606/issn.1806-6976. smad.2018.000366.

15. Moreira WC. Álcool e outras drogas: contribuições de uma liga acadêmica para a formação em Enfermagem. Rev. enferm. UFPI [Internet]. 2017;6(3):83-88. DOI: $10.26694 /$ reufpi.v6i3.6084
16. Oliveira EN, Viana $L S$, Oliveira $L S$, Aragão $H L$, Sousa FiIho AL, Sobrinho NV et al. Liga interdisciplinar em saúde mental: trilhando caminhos para a promoção em saúde. Saúde em Redes. 2019; 5(3):317327. DOI: 10.18310/2 $4464813.2019 v 5 n 3 p 317327$.

17. Silva JVS, Santos Júnior CJ, Ribeiro MC. Ensino integrado em Saúde e prática interprofissional. Rev. Docência Ens. Sup. [Internet].2019;90:1-14. DOI: 10.35699/22375864.2019.14820.

18. Silva JVS, Ribeiro MC. O docente de Enfermagem e sua percepção sobre as ações integrativas na Saúde e na formação interprofissional. Rev. Docência Ens. Sup. [Internet] 2018;8(2):245-61. DOI: 10.35699/22375864.2018.2464.

19. Moraes MMS, Roner MNB, Rocha EMS, Maia RMCS. Interdisciplinaridade e interprofissionalidade. Rev. Docência Ens. Sup. [Internet]. 2019;90:1-17. DOI: 10.35699/2237-5864.2019.13548.

\section{Conflito de Interesses}

não há.

\section{Fonte de fomento}

não há.

Aprovação do comitê de ética em pesquisa: não se aplica.

Registro dos ensaios clínicos em base de acesso público: não se aplica.

Autor Correspondente:

John Victor dos Santos Silva

john.setedejulho@gmail.com

Editor:

Prof. Dr. Paulo Henrique Manso

Recebido: $26 / 08 / 2020$

Aprovado: 24/11/2020 